\title{
Specific protein biomarker patterns for Alzheimer's disease: improved diagnostics in progress
}

\author{
Illana Gozes ${ }^{1}$ (D) \\ Received: 14 July 2017 / Accepted: 10 August 2017 /Published online: 4 September 2017 \\ (C) European Association for Predictive, Preventive and Personalised Medicine (EPMA) 2017
}

\begin{abstract}
This short review looks at Alzheimer's disease (AD) diagnosis through my own point of view, going from imaging through cerebrospinal fluid to blood proteins. Over the last couple of years, we have published two papers targeted at Alzheimer's diagnosis. In one paper, we took an approach of selecting a specific target, namely, activitydependent neuroprotective protein (ADNP), and our results tightened the association of ADNP blood expression with intelligence. In another paper, we took an unbiased approach of analysis of all genes expressed in lymphoblastoid cells lines and discovered changes in expression of the regulator of G-protein signaling 2 (RGS2) as a potential $\mathrm{AD}$ predictor. This review will assess our data in comparison to selected independent studies focusing on blood protein biomarkers as well as assessing saliva and urine samples with potential predictive value for AD. Furthermore, the review will provide directions for a combination of innovative markers, stratifying the population toward disease prevention and personalized medicine.
\end{abstract}

Keywords Predictive $\cdot$ Preventive $\cdot$ Personalized medicine Alzheimer's disease (AD)

\section{Illana Gozes}

igozes@post.tau.ac.il

1 The Lily and Avraham Gildor Chair for the Investigation of Growth Factors, Elton Laboratory for Neuroendocrinology, Department of Human Molecular Genetics and Biochemistry, Sackler Faculty of Medicine, Adams Super Center for Brain Studies and Sagol School of Neuroscience, Tel Aviv University, 69978 Tel Aviv, Israel

\section{AD onset and progression, imaging of protein biomarkers and cerebrospinal fluid analysis}

In an article cited $>2000$ times, Jack and colleagues outlined the dynamic progression of $\mathrm{AD}$, starting from the accumulation of amyloid beta plaques, continuing with tau pathology, followed by changes in brain structure, then memory loss, and finally accumulation of clinical deterioration [1]. This article is coupled with another article entitled multi-modal techniques for diagnosis and prognosis of Alzheimer's disease [2] and the timing of appearance of tau versus amyloid toxicity/pathology is still under investigation.

While imaging of $\mathrm{AD}$ amyloid beta plaques is commercially available since August 2014 (https://www.itnonline.com/ content/first-us-commercial-neuraceq-scan-beta-amyloidplaque-imaging-performed-wvu-healthcare), future studies are required regarding beta-amyloid thresholds associated with the first signs of accelerating rates of cognitive decline [3, 4].

I have extensively reviewed tau pathology for predictive diagnostics, targeted preventive and personalized medicine and application of advanced research in medical practice [5]. As the field is progressing, we have further recently reviewed the status of tau imaging (Höglund, Höglinger, Quinn, Hooper, and Gozes, submitted for publication). In short, $\left[{ }^{18} \mathrm{~F}\right] \mathrm{AV} 1451$ used to label tau tangles showed a significant correlation between the degree of regional MRI brain atrophy and the extent of binding [6]. However, $\left[{ }^{18} \mathrm{~F}\right] \mathrm{AV} 1451$ interacts also with neuromelanin [7] and shows differences between $\mathrm{AD}$ and progressive supranuclear palsy (PSP) [8]. Thus, the interpretation of $\left[{ }^{18} \mathrm{~F}\right]$ THK5351 PET images, with respect to tau, is confounded by the high MAO-B availability across the entire brain [9, $10]$, paving the path to future studies with the recent development of Merck's MK-6240 and Piramal's PI-2620.

As brain imaging is expensive, cerebrospinal fluid (CSF) biomarkers are further explored including, but not limited to 
amyloid beta and tau. In this respect, $\mathrm{AD}$ presents a decrease of CSF amyloid- $\beta_{42}\left(A \beta_{42}\right)$ burden and an increase in cerebrospinal fluid total tau (T-tau). Genome-wide association study (GWAS) and interaction study of $\mathrm{T}$-tau/ $\mathrm{A} \beta_{42}$ robustly replicated previously reported AD-related genes APOE4, APOC1, and TOMM40 and suggested other influencing factors, warranting future investigation [11]. Focusing on phosphorylated tau, GWAS identified a panel of five SNPs, rs6766238, rs1143960, rs1249963, rs11975968, and rs4836493, that are predictive for p-tau181/A $\beta 1-42$ ratio (high/low), suggesting a panel of SNPs as a potential prognostic biomarker in ApoE4negative MCI patients [12]. There is also much interest in the use of CSF fragments of the tau endopeptidom [13] as biomarkers, with the asparagine endopeptidase mediated-NTF, tau $^{1-368}$ [14] potentially acting as an AD specific biomarker. However, CSF biomarker analysis is also invasive and the question is if blood or even saliva can be of use.

\section{Body fluids biomarkers}

As imaging technologies are expensive, somewhat cumbersome, exposing the brain and body at times to radioactive compound and not readily accessible to the general public on a daily basis, and as CSF sampling is relatively invasive, it is of interest to develop minimally invasive predictive and preventive measurements. Blood biomarkers present minimal invasiveness, saliva and urine biomarkers are the least invasive and should be borne in mind for future predictive, preventive and personalized medicine, toward precision in medical practice.

\section{Blood biomarkers}

An original work in 2007 looked at plasma proteomics and claimed to identify 18 signaling proteins that could potentially classify blinded samples from AD and control subjects including ANG-2, angiopoietin-2. CCL, chemokine that contains a C-C motif; CXCL, chemokine that contains a C-X-C motif; G-CSF, granulocyte-colony stimulating factor; GDNF, glialderived neurotrophic factor; ICAM-1, intercellular adhesion molecule-1; IGFBP-1, insulin-like growth factor-binding protein-6; IL, interleukin; PDGF-BB, platelet-derived growth factor BB; TRAIL-R4, TNF-related apoptosis-inducing ligand receptor-4 [15]. Some of these results were verified independently, showing an increased concentration in Biocoll isolated plasma of 5 out of these proteins in $\mathrm{MCI}$ and $\mathrm{AD}$ patients compared to healthy subjects: EGF, GDNF and MIP1 (in AD), MIP4 (in MCI), and RANTES (in MCI and AD) [16].

A relatively recent work looked at complete proteomics searching for blood-based biomarker candidates of cerebral amyloid using PiB (amyloid) PET in non-demented elderly discovering seven plasma proteins, including A2M, Apo-A1, and multiple complement proteins, as pre-clinical biomarkers of amyloid burden, toward early diagnosis [17]. Another work collected human serum specimens from 45 early-stage $A D$ patients (mean age 77.2 years) and 20 normal individuals (mean age 72.3 years) identified by similar technologies 5 up-regulated proteins, namely, actin, apolipoprotein A-IV (Apo A-IV), inter-alpha-trypsin inhibitor heavy chain $\mathrm{H} 4$ (ITIH4), alpha-1-antitrypsin (AAT), and antithrombin-III (AT-III); and one down-regulated protein, activity-dependent neuroprotecting protein (ADNP) in early AD patients [18]. Interestingly, not the same proteins were picked up as major blood players in the two studies cited above, suggesting potential impact for the disease status, non-demented elderly exhibiting amyloid plaques vs. early $\mathrm{AD}$.

Discovered in our laboratory [19, 20], ADNP is essential for brain formation [21], and ADNP deficits in mice results in tau pathology coupled to cognitive impairments [22]. In this respect, de novo, mostly truncating mutations in ADNP lead to cognitive impairments and autism in children [23-26]. Importantly, we have recently shown that serum ADNP content [27] is correlated with premorbid intelligence. Thus, age adjustment showed significant associations between higher premorbid intelligence and greater serum ADNP, in agreement with the results described above. Furthermore, greater cortical amyloid correlated with lower ADNP and its family member ADNP2 blood-borne mRNAs. Significant increases in lymphocyte ADNP mRNA levels were observed in patients ranging from mild cognitive impairment (MCI) to $\mathrm{AD}$ dementia. ADNP is suggested as a novel biomarker toward screening and tracking $\mathrm{AD}$.

Taking a different approach, we applied genome-wide transcriptomic profiling of lymphoblastoid cells lines (LCLs) from healthy individuals and $\mathrm{AD}$ patients for identifying genes that predict sensitivity to $A \beta$. Lower expression of RGS2 (regulator of G-protein signaling 2), a key control element of Gprotein-coupled receptor signaling, was discovered compared to controls as listed below: (1) LCLs from healthy individuals exhibiting high vs. low A $\beta$ sensitivity. (2) AD LCLs correlated with cognitive function. (3) Postmortem AD brain tissues. (4) $\mathrm{MCI}$ and AD blood samples. RGS2 is also suggested as a novel AD biomarker (alongside other genes), [28].

The differences observed in the potential protein markers identified attests to differential protein synthesis and protein stability in plasma, serum and blood cells, requiring a specific routine and critical requirements as implemented by large consortia like ADNI [29]. Furthermore, the possibility of personalized stratification is also tantalizing. Regardless, looking at ADNP in two geographically/ethically different groups, in one paper from Taiwan [18] and in one paper including a collaborative work between the USA and Israel [27], correlated serum ADNP levels with cognition/AD. 
Current and future studies are also looking at blood exosomes [30]. Targeted measurements of proteins revealed that the levels of autolysosomal proteins in neurally derived blood exosomes distinguish patients with $\mathrm{AD}$ from controls, reflecting the pathology of $\mathrm{AD}$ years before clinical onset. These proteins includes cathepsin D, lysosome-associated membrane protein 1 (LAMP-1), and ubiquitinylated proteins. In this respect, it is interesting to add that ADNP regulates cathepsin expression [31]. ADNP was further associated with autophagy [32]. Developing an algorithm that will take into account variations and relationships among the potential biomarkers listed above and beyond will bring us closer to precision medicine.

\section{Saliva biomarkers}

While blood serves a minimally invasive source for surrogate biomarkers, other body fluids, which do not require any body invasiveness include, for example, saliva. Recent comprehensive reviews on AD biomarkers exist [33], and specifically about saliva, suggesting early diagnosis of $\mathrm{MCI}$ and $\mathrm{AD}$ based on salivary lactoferrin, an iron- and also $\mathrm{A} \beta$-binding glycoprotein [34].

In contrast, it is worth mentioning that the activity of salivary acetylcholinesterase $(\mathrm{AChE})$, which has been proposed as a potential biochemical marker of cholinergic function associated with $\mathrm{AD}$, only trended toward reduction in the $\mathrm{AD}$ cohort compared to the control group and this apparent reduction was insignificant [35].

It should be noted that the literature on saliva biomarkers for AD is still very limited (42 articles are currently available on PUBMED for saliva and Alzheimer's), however, for the purpose of this review and future improved diagnostics it is worth mentioning some, as follows.

As a starting point, saliva is believed to be essential for the preservation of oral health and function, thus, unstimulated and stimulated submandibular salivary gland secretions were collected from non-medicated and otherwise healthy patients suffering from dementia and of the AD type (early disease stages) and compared to age-matched healthy controls. Results showed significantly lower salivary flow rates in the dementia sufferers, suggestive of a selective impairment in submandibular gland function in essentially healthy patients with early-stage dementia [36]. In this respect, cortisol can also be measured in saliva linking to stress/anxiety and depression as opposed to well-being, risks and protectors, respectively in terms of AD [37]. Furthermore, Dehydroepiandrosterone (DHEA) can be measured as well and correlated with cortisol [38].

In general related to body fluids, future studies are warranted combining proteomics, metabolomics [39] and genomic evaluations with large cohorts.

\section{Urine biomarkers}

Like in the saliva, urinary free cortisol (UFC) and creatinine (Cr) were measured, and a UFC/Cr ratio was calculated at a personal level showing that UFC/Cr level and UFC/Cr variability were significant predictors of $\mathrm{AD}$ risk an average of 2.9 years before $\mathrm{AD}$ onset. It was thus suggested that cortisol dysregulation as above may modulate the downstream clinical expression of $\mathrm{AD}$ pathology or present a preclinical marker for $\mathrm{AD}[40]$.

However, urine samples are not used for AD diagnosis, although those might be attractive for a relatively noninvasive sample collection. Previous studies have indicated some other utility for such samples, e.g., assessing environmental hazards, such as cadmium [41] and measuring oxidative stress markers such as F4-neuroprostanes, F3neuroprostanesn-6 DPA, and F2-dihomo-isoprostanes, metabolites of non-enzymatic lipid peroxidation of polyunsaturated fatty acids [docosahexaenoic acid, n-6 docosapentanoic acid, and adrenic acid, respectively] [42]. Importantly, these studies await robust clinical validations [43] and protein biomarkers have yet to be evaluated in depth.

\section{Expert recommendations and outlook}

In summary, here, we contribute to further understanding of biomarkers that can facilitate early diagnosis, prediction, classification/patient stratification, prognosis, and potentially treatment response, which could be used in clinical practice in line with follow-up steps in AD-dedicated research to satisfy patients' needs and to advance healthcare as elegantly outlined before for other diseases and for disease states in general [44].

We focus here on proteins, broadly speaking proteomics, the study of the entire population of proteins and peptides in an organism or a part of it, such as a cell, tissue, or fluids like CSF, plasma, serum, urine, or saliva. We also discuss some transcriptomics. The proteomics field has been reviewed for AD describing methodology that was applied in $>100$ studies, extracting a list of 366 proteins and peptides as potential targets in $\mathrm{AD}$ [45]. The current short review adds complexity to the list with more recent publications, leading to an outlook for future examinations and direct recommendations.

The next steps to be performed in order to advance the healthcare in the area should include additional advanced proteomics as well as bioinformatics mining and modeling methods for the identification of disease mechanisms in $\mathrm{AD}$ [46]. Future studies are also looking at the microbiome, an emerging active field of research [47]. The identification of disease mechanisms allows the development of novel biomarkers, targeted at disease prevention, monitoring and halting disease progression and drug responsiveness toward disease modification. Large consortia like ADNI [11;29] will 
facilitate these important studies toward personalized, precision medicine.

Funding This study was funded by the Lily and Avraham Gildor Chair for the Investigation of Growth Factors, Elton Laboratory for Neuroendocrinology and the following grants, ISF 1424/14, ERA-NET neuron AUTYSM, AMN Foundation as well as Drs. Ronith and Armand Stemmer and Mr. Arthur Gerbi (French Friends of Tel Aviv University), and Canadian and Spanish Friends of Tel Aviv University.

\section{Compliance with ethical standards}

Conflict of interest Professor Illana Gozes serves as the Chief Scientific Officer of Coronis Neurosciences.

\section{References}

1. Jack CR Jr, Knopman DS, Jagust WJ, Shaw LM, Aisen PS, Weiner MW, et al. Hypothetical model of dynamic biomarkers of the Alzheimer's pathological cascade. Lancet Neurol. 2010;9:119-28.

2. Perrin RJ, Fagan AM, Holtzman DM. Multimodal techniques for diagnosis and prognosis of Alzheimer's disease. Nature. 2009;461: 916-22.

3. Snitz BE, Lopez OL, McDade E, Becker JT, Cohen AD, Price JC, et al. Amyloid-beta imaging in older adults presenting to a memory clinic with subjective cognitive decline: a pilot study. J Alzheimers Dis. 2015;48(Suppl 1):S151-9.

4. P.S. Insel, N. Mattsson, R.S. Mackin, M. Scholl, R.L. Nosheny, D. Tosun, M.C. Donohue, P.S. Aisen, W.J. Jagust, M.W. Weiner, and I. Alzheimer's disease neuroimaging, accelerating rates of cognitive decline and imaging markers associated with beta-amyloid pathology. Neurology 86 (2016) 1887-96.

5. Gozes I. Tau pathology: predictive diagnostics, targeted preventive and personalized medicine and application of advanced research in medical practice. EPMA J. 2010;1:305-16.

6. Spina S, Schonhaut DR, Boeve BF, Seeley WW, Ossenkoppele R, O'Neil JP, et al. Frontotemporal dementia with the V337M MAPT mutation: Tau-PET and pathology correlations. Neurology. 2017;88:758-66.

7. Hansen AK, Knudsen K, Lillethorup TP, Landau AM, Parbo P, Fedorova T, et al. In vivo imaging of neuromelanin in Parkinson's disease using 18F-AV-1451 PET. Brain. 2016;139:2039-49.

8. Passamonti L, Vazquez Rodriguez P, Hong YT, Allinson KS, Williamson D, Borchert RJ, et al. 18F-AV-1451 positron emission tomography in Alzheimer's disease and progressive supranuclear palsy. Brain. 2017;140:781-91.

9. Chiotis K, Saint-Aubert L, Rodriguez-Vieitez E, Leuzy A, Almkvist O, Savitcheva I, et al. Longitudinal changes of tau PET imaging in relation to hypometabolism in prodromal and Alzheimer's disease dementia. Mol Psychiatry. 2017;

10. Ng KP, Pascoal TA, Mathotaarachchi S, Therriault J, Kang MS, Shin M, et al. Monoamine oxidase B inhibitor, selegiline, reduces 18F-THK5351 uptake in the human brain. Alzheimers Res Ther. 2017;9:25.

11. Li J, Zhang Q, Chen F, Meng X, Liu W, Chen D, et al. Genomewide association and interaction studies of CSF T-tau/Abeta42 ratio in ADNI cohort. Neurobiol Aging. 2017;57:247.e1-8.

12. Sun Y, Bresell A, Rantalainen M, Hoglund K, Lebouvier T, Salter $\mathrm{H}$, et al. An integrated bioinformatics approach for identifying genetic markers that predict cerebrospinal fluid biomarker p-tau181/ Abeta1-42 ratio in ApoE4-negative mild cognitive impairment patients. J Alzheimers Dis. 2015;45:1061-76.
13. Hansson KT, Skillback T, Pernevik E, Kern S, Portelius E, Hoglund $\mathrm{K}$, et al. Expanding the cerebrospinal fluid endopeptidome. Proteomics. 2017. https://doi.org/10.1002/pmic.201600384.

14. Zhang Z, Song M, Liu X, Kang SS, Kwon IS, Duong DM, et al. Cleavage of tau by asparagine endopeptidase mediates the neurofibrillary pathology in Alzheimer's disease. Nat Med. 2014;20:125462.

15. Ray S, Britschgi M, Herbert C, Takeda-Uchimura Y, Boxer A, Blennow K, et al. Classification and prediction of clinical Alzheimer's diagnosis based on plasma signaling proteins. Nat Med. 2007;13:1359-62.

16. Marksteiner J, Kemmler G, Weiss EM, Knaus G, Ullrich C, Mechtcheriakov S, et al. Five out of 16 plasma signaling proteins are enhanced in plasma of patients with mild cognitive impairment and Alzheimer's disease. Neurobiol Aging. 2011;32:539-40.

17. Westwood S, Leoni E, Hye A, Lynham S, Khondoker MR, Ashton NJ, et al. Blood-based biomarker candidates of cerebral amyloid using PiB PET in non-demented elderly. J Alzheimers Dis. 2016;52:561-72.

18. Yang MH, Yang YH, Lu CY, Jong SB, Chen LJ, Lin YF, et al. Activity-dependent neuroprotector homeobox protein: a candidate protein identified in serum as diagnostic biomarker for Alzheimer's disease. J Proteome. 2012;75:3617-29.

19. Bassan M, Zamostiano R, Davidson A, Pinhasov A, Giladi E, Perl $\mathrm{O}$, et al. Complete sequence of a novel protein containing a femtomolar-activity-dependent neuroprotective peptide. J Neurochem. 1999;72:1283-93.

20. Zamostiano R, Pinhasov A, Gelber E, Steingart RA, Seroussi E, Giladi E, et al. Cloning and characterization of the human activitydependent neuroprotective protein. J Biol Chem. 2001;276:708-14.

21. Pinhasov A, Mandel S, Torchinsky A, Giladi E, Pittel Z, Goldsweig $\mathrm{AM}$, et al. Activity-dependent neuroprotective protein: a novel gene essential for brain formation. Brain Res Dev Brain Res. 2003;144: 83-90.

22. Vulih-Shultzman I, Pinhasov A, Mandel S, Grigoriadis N, Touloumi O, Pittel Z, et al. Activity-dependent neuroprotective protein snippet NAP reduces tau hyperphosphorylation and enhances learning in a novel transgenic mouse model. J Pharmacol Exp Ther. 2007;323:438-49.

23. Gozes I, Helsmoortel C, Vandeweyer G, Van der Aa N, Kooy F, Sermone SB. The compassionate side of neuroscience: Tony Sermone's undiagnosed genetic journey-ADNP mutation. J Mol Neurosci. 2015;56:751-7.

24. Gozes I, Patterson MC, Van Dijck A, Kooy RF, Peeden JN, Eichenberger JA, et al. The eight and a half year journey of undiagnosed $\mathrm{AD}$ : gene sequencing and funding of advanced genetic testing has led to hope and new beginnings. Front Endocrinol. 2017;8:107.

25. Gozes I, Van Dijck A, Hacohen-Kleiman G, Grigg I, Karmon G, Giladi E, et al. Premature primary tooth eruption in cognitive/ motor-delayed ADNP-mutated children. Transl Psychiatry. 2017;7:e1043.

26. Helsmoortel C, Vulto-van Silfhout AT, Coe BP, Vandeweyer G, Rooms L, van den Ende J, et al. A SWI/SNF-related autism syndrome caused by de novo mutations in ADNP. Nat Genet. 2014;46: $380-4$

27. Malishkevich A, Marshall GA, Schultz AP, Sperling RA, AharonPeretz J, Gozes I. Blood-borne activity-dependent neuroprotective protein (ADNP) is correlated with premorbid intelligence, clinical stage, and Alzheimer's disease biomarkers. J Alzheimers Dis. 2016;50:249-60.

28. Hadar A, Milanesi E, Squassina A, Niola P, Chillotti C, PasmanikChor M, et al. RGS2 expression predicts amyloid-beta sensitivity. MCI and Alzheimer's disease: genome-wide transcriptomic profiling and bioinformatics data mining. Transl Psychiatry. 2016;6: e909. 
29. Wang ML, Wei XE, Yu MM, Li PY, Li WB et al. Self-reported traumatic brain injury and in vivo measure of AD-vulnerable cortical thickness and AD-related biomarkers in the ADNI cohort. Neurosci Lett. 2017;655:115-20.

30. Goetzl EJ, Boxer A, Schwartz JB, Abner EL, Petersen RC, Miller $\mathrm{BL}$, et al. Altered lysosomal proteins in neural-derived plasma exosomes in preclinical Alzheimer disease. Neurology. 2015;85: 40-7.

31. Mandel S, Rechavi G, Gozes I. Activity-dependent neuroprotective protein (ADNP) differentially interacts with chromatin to regulate genes essential for embryogenesis. Dev Biol. 2007;303:814-24.

32. Merenlender-Wagner A, Malishkevich A, Shemer Z, Udawela M, Gibbons A, Scarr E, et al. Autophagy has a key role in the pathophysiology of schizophrenia. Mol Psychiatry. 2015;20:126-32.

33. Sharma S, Lipincott W. Biomarkers in Alzheimer's disease-recent update. Curr Alzheimer Res. 2017. https://doi.org/10.2174/ 1567205014666170220141822.

34. Carro E, Bartolome F, Bermejo-Pareja F, Villarejo-Galende A, Molina JA, Ortiz P, et al. Early diagnosis of mild cognitive impairment and Alzheimer's disease based on salivary lactoferrin. Alzheimers Dement. 2017;8:131-8.

35. Bakhtiari S, Moghadam NB, Ehsani M, Mortazavi H, Sabour S, Bakhshi M. Can salivary acetylcholinesterase be a diagnostic biomarker for Alzheimer? J Clin Diagn Res. 2017;11:ZC58-60.

36. Ship JA, DeCarli C, Friedland RP, Baum BJ. Diminished submandibular salivary flow in dementia of the Alzheimer type. J Gerontol. 1990;45:M61-6.

37. Wahbeh H, Kishiyama SS, Zajdel D, Oken BS. Salivary cortisol awakening response in mild Alzheimer disease, caregivers, and noncaregivers. Alzheimer Dis Assoc Disord. 2008;22:181-3.

38. Correa MS, Vedovelli K, Giacobbo BL, de Souza CE, Ferrari P, de Lima A, et al. Psychophysiological correlates of cognitive deficits in family caregivers of patients with Alzheimer disease. Neuroscience. 2015;286:371-82.
39. Yilmaz A, Geddes T, Han B, Bahado-Singh RO, Wilson GD, Imam $\mathrm{K}$, et al. Diagnostic biomarkers of Alzheimer's disease as identified in saliva using $1 \mathrm{H}$ NMR-based metabolomics. J Alzheimers Dis. 2017;58:355-9.

40. Ennis GE, An Y, Resnick SM, Ferrucci L, O'Brien RJ, Moffat SD. Long-term cortisol measures predict Alzheimer disease risk. Neurology. 2017;88:371-8.

41. Peng Q, Bakulski KM, Nan B, Park SK. Cadmium and Alzheimer's disease mortality in U.S. adults: updated evidence with a urinary biomarker and extended follow-up time. Environ Res. 2017;157: 44-51.

42. De Las Heras-Gomez I, Medina S, Casas-Pina T, Marin-Soler L, Tomas A, Martinez-Hernandez P, et al. Potential applications of lipid peroxidation products - F4-neuroprostanes, F3neuroprostanesn-6 DPA, F2-dihomo-isoprostanes and F2isoprostanes - in the evaluation of the allograft function in renal transplantation. Free Radic Biol Med. 2017;104:178-84.

43. Garcia-Blanco A, Baquero M, Vento M, Gil E, Bataller L, ChaferPericas C. Potential oxidative stress biomarkers of mild cognitive impairment due to Alzheimer disease. J Neurol Sci. 2017;373:295302 .

44. Golubnitschaja O, Baban B, Boniolo G, Wang W, Bubnov R, Kapalla M, et al. Medicine in the early twenty-first century: paradigm and anticipation - EPMA position paper 2016. EPMA J. 2016;7:23.

45. Zurbig P, Jahn H. Use of proteomic methods in the analysis of human body fluids in Alzheimer research. Electrophoresis. 2012;33:3617-30.

46. Hofmann-Apitius M, Ball G, Gebel S, Bagewadi S, de Bono B, Schneider R, et al. Bioinformatics mining and modeling methods for the identification of disease mechanisms in neurodegenerative disorders. Int J Mol Sci. 2015;16:29179-206.

47. Alkasir R, Li J, Li X, Jin M, Zhu B. Human gut microbiota: the links with dementia development. Protein Cell. 2017;8:90-102. 